\title{
Cortical microstimulation in auditory cortex of rat elicits best-frequency dependent behaviors
}

\author{
Kevin J Otto ${ }^{1,3}$, Patrick J Rousche ${ }^{2}$ and Daryl R Kipke ${ }^{1}$ \\ ${ }^{1}$ Department of Biomedical Engineering, University of Michigan, Ann Arbor, MI 48109-2125, USA \\ ${ }^{2}$ Department of Bioengineering, University of Illinois at Chicago, Chicago, IL 60607, USA \\ E-mail: dkipke@umich.edu
}

Received 7 December 2004

Accepted for publication 7 April 2005

Published 31 May 2005

Online at stacks.iop.org/JNE/2/42

\begin{abstract}
Electrical activation of the auditory cortex has been shown to elicit an auditory sensation; however, the perceptual effects of auditory cortical microstimulation delivered through penetrating microelectrodes have not been clearly elucidated. This study examines the relationship between electrical microstimulus location within the adult rat auditory cortex and the subsequent behavior induced. Four rats were trained on an auditory frequency discrimination task and their lever-pressing behavior in response to stimuli of intermediate auditory frequencies was quantified. Each trained rat was then implanted with a microwire array in the auditory cortex of the left hemisphere. Best frequencies (BFs) of each electrode in the array were determined by both local field potential and multi-unit spike-rate activity evoked by pure tone stimuli. A cross-dimensional psychophysical generalization paradigm was used to evaluate cortical microstimulation-induced behavior. Using the BFs of each electrode, the microstimulation-induced behavior was evaluated relative to the auditory-induced behavior. Microstimulation resulted in behavior that was dependent on the BFs of the electrodes used for stimulation. These results are consistent with recent reports indicating that electrophysiological recordings of neural responses to sensory stimuli may provide insight into the sensation generated by electrical stimulation of the same sensory neural tissue.
\end{abstract}

\section{Introduction}

A set of now-classical studies introduced the use of electrical stimulation of human sensory cortex to elicit sensory perceptions (Brindley and Lewin 1968a, Dobelle et al 1973, Penfield and Rasmussen 1950). While groundbreaking, these studies were limiting in terms of the relatively high stimulation currents and unreliable percepts that resulted, in part, from the use of surface electrodes for epicortical stimulation. The use of penetrating intracortical microelectrodes addresses the high stimulation level issue by bringing the sites of activation closer to the target neuronal population to result in lower stimulation thresholds and more confined stimulus volumes

\footnotetext{
3 Current address: Kresge Hearing Research Institute, Department of
} Otolaryngology, University of Michigan, MI, USA.
(Bartlett and Doty 1980, Ronner et al 1981, Stoney et al 1968). More detailed investigation of the efficacy of intracortical microstimulation to transmit sensory information directly to sensory cortex is driven by developments in microelectrode technologies, which now provide a means to establish chronic intracortical neural interfaces having precise two- or threedimensional arrangement of stimulating sites.

Cortical microstimulation can be utilized to provide a 'virtual' signal that subjects use in the processing of local information. Cortical stimulation of sensorimotor areas can be utilized as a conditional stimulus in a classical conditioning paradigm (Woody and Engel 1972). Newsome and colleagues have shown that a primate's perceptual decisions can be biased by microstimulation of the middle temporal visual cortical area (Groh et al 1997, Salzman et al 1990, 1992, Seidemann et al 1998). Recent studies by Romo and 


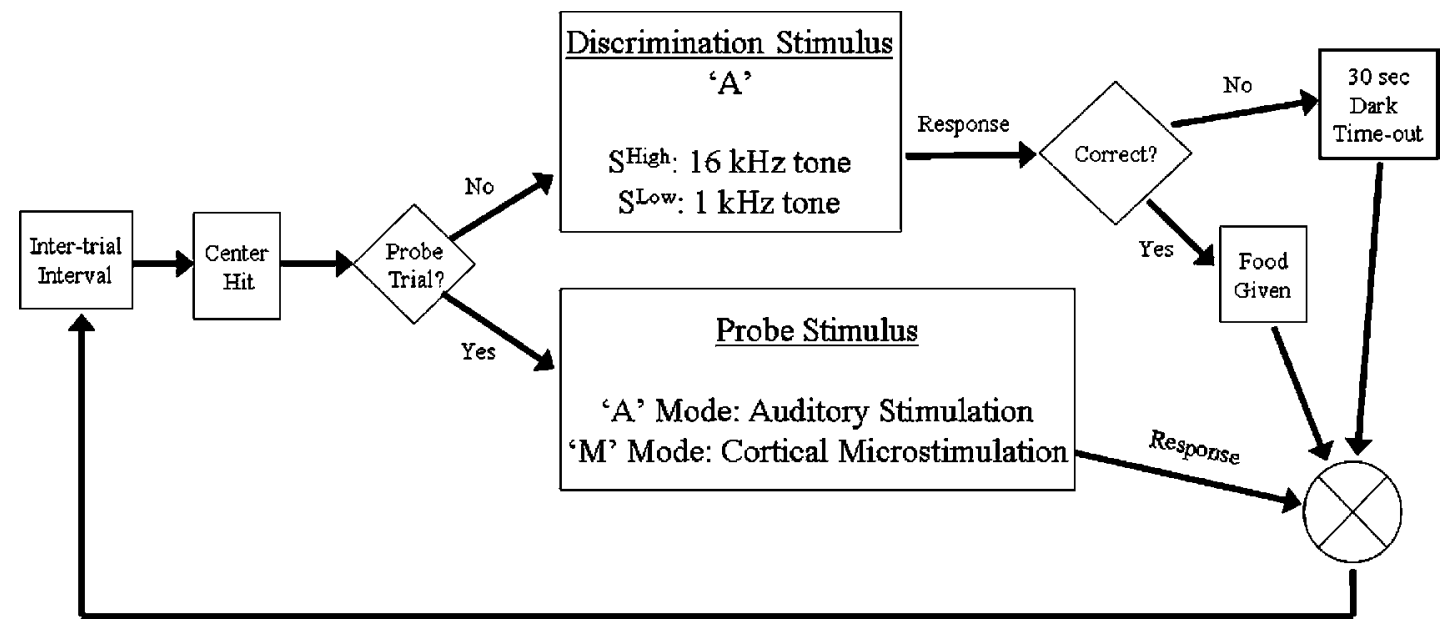

Figure 1. Trial behavioral flowchart. Each daily testing session consisted of approximately 275 trials. The context of the trial presentations randomly varied; $75 \%$ were discrimination trials (auditory task), and the remaining $25 \%$ were probe trials. On a discrimination trial, correct auditory tone identification of either $16 \mathrm{kHz}$ or $1 \mathrm{kHz}$ was rewarded by a $4.5 \mathrm{mg}$ food pellet; incorrect identification was reinforced by a $30 \mathrm{~s}$ dark time-out. During probe trials, the subject was presented an atypical stimulus of one of two modes. The 'A' mode recorded the subject's responses to tones of intermediate auditory frequencies. The ' $M$ ' mode recorded the subject's responses to electrical microstimulation in the auditory cortex. After subject responses to the probe stimuli were recorded, the next trial was initiated; the probe trials were never reinforced. Only one probe mode was used in a given testing session, resulting in two possible sets of behavioral data, A-A (auditory task, auditory probe) and A-M (auditory task, microstimulation probe).

colleagues have shown that microstimulation of the primate somatosensory cortex using putative temporal stimulation parameters provides salient sensory information to behaving primates (Romo et al 2000, 1998). In order to further understand cortical neural coding for the development of cortical neuroprostheses, elucidation of both the properties of the electrical stimulus as well as encoding strategies to increase the information capacity of cortical microstimulation are required.

Previous studies have shown that that Mongolian gerbils (Scheich and Breindl 2002), cats (Rousche and Normann 1999) and rats (Rousche et al 2003) are able to detect microstimulation of auditory cortex. Further, Rousche et al showed that cortical microstimulation of increasing intensity was more readily detected. This result paralleled auditory tone intensity detection behavior. Interestingly, the detection results were similar regardless of the stimulus location within the auditory cortex, even though the rat primary auditory cortex is tonotopically organized (Sally and Kelly 1988); however, since a detection paradigm was used, the spectral-based sensation effects of stimulus location could not be determined. Human studies have shown that surface stimulation of human primary auditory cortex evokes perceptions that contain a pitch (Dobelle et al 1973, Penfield and Perot 1963). The pitch was reported to depend on electrode location within the primary auditory field, which was theorized to be attributed to the arrangement of iso-frequency bands into a tonotopic map.

The objective of this study was to determine whether microstimulation within iso-frequency bands in the primary auditory cortex would elicit behavior that was consistent with that elicited by the corresponding auditory tone. A cross-dimensional psychophysical task was used to determine the relative sensations of auditory and cortical stimulation in the adult white rat. Cortical microstimulation elicited behaviors relative to the best frequency $(\mathrm{BF})$ of the electrode location that were correlated with behavior evoked by acoustic stimulation of the same frequency. These results demonstrate that spatially distributed microstimulation in auditory cortex provides 'virtual' auditory cues with distinct spectral content.

\section{Methods}

\section{Behavioral training}

Four male, naïve Sprague-Dawley rats (250-300 g) were trained in an auditory discrimination task. Initially, the rats were food deprived to $80 \%$ of their free-feeding weight. Subjects were generally trained 5-6 days a week, and given food on off days to maintain their food deprivation weight. Subjects responded in standard operant conditioning behavioral boxes (Med Associates, St Albans, VT) located within an anechoic room. The response wall of the test box included three side-by-side retractable response levers approximately 4 inches above the cage floor. A house light at the rear of the box was utilized for both illumination and negative reinforcement. The behavioral apparatus was controlled and monitored by software developed in-house, running on a PC interfaced with digital input-output hardware (System II, Tucker-Davis Technologies, Gainesville, FL). This equipment was also used to generate all auditory stimuli used in the experiment. The auditory stimuli were delivered via a speaker (Yamaha NS-10M Studio, Yamaha Corporation, Buena Park, CA) located $1 \mathrm{~m}$ directly above the test box. The system was calibrated to a position directly above the center lever and delivered a near-flat frequency response between $500 \mathrm{~Hz}$ and $32 \mathrm{kHz}$.

A discrimination task in a forced-choice psychophysical paradigm was used to assess stimulus generalization (figure 1). 

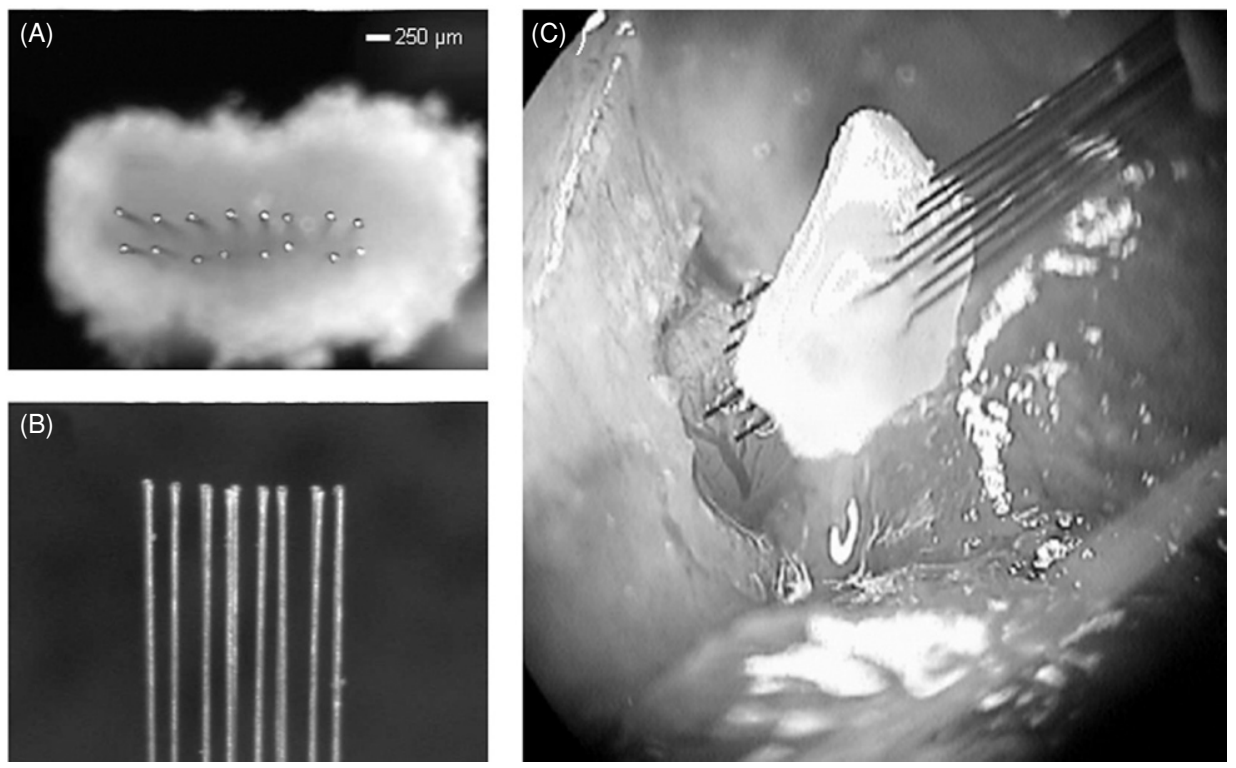

Figure 2. Chronic microwire rat auditory cortex preparation. Arrays of $50 \mu \mathrm{m}$ polyimide-coated tungsten microwires were assembled into an array consisting of two rows of eight electrodes. Electrode centers were spaced approximately $250 \mu \mathrm{m}$ apart. (A) Top view of a sample electrode array viewed from the tip. (B) Side view of the same electrode array. (C) Electrode array just prior to insertion into left hemisphere auditory cortex. The electrode array insertion site was first located stereotaxically, and subsequently confirmed via vascular landmarks. The array was positioned to avoid major vascular injury and implanted between the large anterior and posterior dorsoventral vessels that characterize the primary auditory cortex in the rat.

Subjects were positively reinforced via single food pellets (P J Noyes, $45 \mathrm{mg}$ rodent diet I, Lancaster, $\mathrm{NH}$ ) for correct responses to trains of auditory stimuli. Initially, all three levers were retracted, and the house light was illuminated. The subjects were signaled to start a single trial by the extension of the center lever. In order to minimize spurious trial initiations, trials were subject-initiated by two recorded presses of this center response lever. Subsequently, the center lever was retracted, and a train ( $250 \mathrm{~ms}$ on, $250 \mathrm{~ms}$ off) of five puretone bursts was delivered. Stimulus trains were utilized instead of long stimulus durations in order to limit the total stimulus duration for the later microstimulation experiments. Auditory training stimuli trains at either $1 \mathrm{kHz}$, or $16 \mathrm{kHz}$ were delivered at $70 \mathrm{~dB}$ SPL. Upon completion of the auditory stimulus presentation, the two outer levers were extended. A fixed-ratio response paradigm was utilized, and in order to stabilize impulsive behavior, subjects were reinforced only after four responses on a given lever within $7 \mathrm{~s}$ of outer lever presentation. Responses were designated correct and positively reinforced for a left-hand lever response to the 1 $\mathrm{kHz}$ stimulus or a right-hand lever response to the $16 \mathrm{kHz}$ stimulus. Left-hand responses to the $16 \mathrm{kHz}$ stimulus or right-hand responses to the $1 \mathrm{kHz}$ stimulus were designated incorrect and negative reinforcement was given in the form of a 30 s dark timeout. A response was considered null, and negatively reinforced, if the subject did not respond within the $7 \mathrm{~s}$ response window. Null response trials in all of the training or testing were rare (zero for $>95 \%$ of the sessions) and were not used in the behavioral data analysis. The general training paradigm generally required several months of training.

\section{Auditory generalization behavioral testing}

Upon criterion performance of the auditory training paradigm (above 90\% for three consecutive days), psychophysical curves were created to assess auditory generalization behavioral performance. The standard auditory $1 \mathrm{kHz}$ or $16 \mathrm{kHz}$ reinforced stimuli were delivered, indicating leftor right-hand behavioral cues respectively; however, in the generalization-testing paradigm, approximately $25 \%$ of the trials were randomly chosen as 'probe' trials in which stimuli of intermediate auditory frequencies were presented, and the subsequent behavior recorded. The probe trials were never reinforced. The rats' generalization behavior in response to four auditory probe frequencies was tested. The auditory probe frequencies were chosen as tones spaced evenly on a logarithmic scale $(1740 \mathrm{~Hz}, 3030 \mathrm{~Hz}, 5280 \mathrm{~Hz}$ and $9190 \mathrm{~Hz})$. Hereto, this test is referred to as 'A-A' (the standard auditory discrimination task, 'A', coupled with auditory probe testing, 'A'). Daily testing sessions continued until each subject received 200 positive rewards. Due to the random trial nature of the probe presentation in the experiment, the exact number of daily trials varied, but was on average approximately 275 . Daily testing sessions lasted approximately $80 \mathrm{~min}$.

\section{Surgical implantation}

After three consecutive successful auditory generalizationtesting sessions, each rat was chronically implanted with an array of microelectrodes. Electrode array construction and surgical implantation have been described in a previous publication (Williams et al 1999). Briefly, the electrode arrays consisted of two rows of eight, $50 \mu \mathrm{m}$ diameter, polyimide coated tungsten microwires (figures 2(A) and (B)). Electrode 
spacing in a given row and between rows was $250 \mu \mathrm{m}$, resulting in an electrode footprint of $1.75 \mathrm{~mm}$ by $0.25 \mathrm{~mm}$. Literature reports have identified the anterior-posterior extent of rat primary auditory cortex as $1.9 \mathrm{~mm}$ (Kilgard and Merzenich 1999) and $2.5 \mathrm{~mm}$ (Sally and Kelly 1988). Thus, our electrode array was designed to span approximately 70$90 \%$ of primary auditory cortex. Animals were anesthetized with a combination of Ketamine $75.0 \mathrm{mg} \mathrm{kg}^{-1}$, Xylazine $7.5 \mathrm{mg} \mathrm{kg}^{-1}$ and Acepromazine $1.5 \mathrm{mg} \mathrm{kg}^{-1}$. The scalp was removed over the left hemisphere, and a $4 \mathrm{~mm} \times 4 \mathrm{~mm}$ crainiotomy was performed at $4 \mathrm{~mm}$ lateral and $5 \mathrm{~mm}$ posterior to bregma. The dura mater was removed and the tissue moistened with sterile saline. The auditory cortex was located stereotaxically and from vascular landmarks as identified in previous studies (Sally and Kelly 1988). Viewed through a microscope, the electrode array was positioned at the surface of the brain between the large anterior and posterior dorsoventral vessels that have been shown in literature to delineate the auditory cortex in the rat (figure 2(C)). The array was rapidly inserted into cortex until visual pia mater penetration was confirmed. The electrode array was then retracted to the previously defined cortical surface and subsequently lowered to $600 \mu \mathrm{m}$. Gelfoam $\Subset$ was positioned on the brain around the electrodes to serve as a protective barrier, and the array was affixed to stainless steel bone screws in the cranium with polymethyl-methacralate. All procedures complied with the United States Department of Agriculture guidelines for the care and use of laboratory animals and were approved by the Institutional Animal Care and Use Committee.

\section{Cortical microstimulation behavioral testing}

After surgical recovery (approx. 1.5 weeks), the rats were tested to ensure that their behavior to the auditory task was unchanged. In all cases, subject behavior returned to presurgical level in less than two sessions.

In order to assess cortical microstimulation, the method of intermixing probe trials with the standard reinforced auditory discrimination trials was again employed. Daily testing sessions were conducted in an identical manner as the A-A testing sessions. The trials were subject initiated by center lever presses, and the trial stimulation was presented after center lever retraction. Approximately $75 \%$ of the trials were the normal auditory task $1 \mathrm{kHz}$ or $16 \mathrm{kHz}$ cues ('A'), and were reinforced appropriately. The resultant $25 \%$ of the trials were microstimulation probe trials, where the cue was cortical microstimulation delivered through a single electrode ('M'). These data are hereto referred to as 'A-M'. Due to electrode array geometry, daily A-M testing sessions were conducted on only a single row.

Electrodes within an array were labeled such that electrode 1 was the most anterior electrode on the dorsal row. Subsequent electrodes on the dorsal row were labeled through electrode 8. Electrode 9 was labeled as the most anterior electrode on the ventral row and the rest of the electrodes on the ventral row were labeled through electrode 16. Four cortical microstimulation probe stimuli were tested on a given row in order to maintain consistency between the A-M and
A-A testing sessions. On A-M1 sessions the four different probe stimuli were microstimulation delivered through electrodes $1,3,5$ or 7. A-M2 probe stimuli were microstimulation delivered through electrodes $9,11,13$ or 15 .

Microstimulation pulse trains consisted of cathodic first, charge-balanced, biphasic square-wave pulses (250 $\mu$ s pulse width) delivered at $200 \mathrm{~Hz}$ and $68 \mu \mathrm{A}$. This stimulus intensity was chosen for two reasons. First, a calculated estimation of current spread based on parameters reported in the literature led to minimal effective stimulation radii (100 $\mu \mathrm{m}$ ) between neighboring electrodes at $68 \mu \mathrm{A}$ (Nunez 1981, Stoney et al 1968). Second, in several literature references, $68 \mu \mathrm{A}$ was a sufficient microstimulation level to ensure that the stimulus was behaviorally robust (Rousche et al 2001, Tehovnik 1996). A waveform generator (WaveTek, Everett, WA) was used to generate the pulse train, which was delivered through an optical stimulus isolator (A-M Systems, Carlsborg, WA) in constant-current stimulation mode. The cortical microstimulation stimulus intensity was confirmed using a $1 \mathrm{k} \Omega$ resistor circuit prior to testing. The cranial stainless-steel screws served as the stimulation return pathway.

The temporal parameters of the microstimulation were chosen to mimic the temporal envelope of the auditory stimuli. Microstimulation pulse trains were delivered in five bursts ( $250 \mathrm{~ms}$ on, $250 \mathrm{~ms}$ off). The behavioral apparatus software recorded responses to both the task stimuli and the probe stimuli. The microstimulation-evoked behavior was evaluated based on BFs of the electrodes as discussed below.

\section{Cortical microstimulation control}

A control experiment was conducted to ensure that no environmental behavioral cues were affecting behavioral discrimination. In the control experiment the subject was reinforced for correct discrimination of the standard auditory task. Session probe stimuli were constructed with the same parameters and probability as the previous A-M sessions; however, in this experiment the stimulus isolator was turned off, ensuring that the animal would not receive cortical microstimulation. Behavioral responses to both the normal auditory discrimination trials and the control probe trials were tabulated and analyzed.

\section{Electrophysiological recording and analysis}

Electrophysiological recordings in response to auditory stimulation were conducted under anesthesia using the aforementioned ketamine/xylazine/acepromazine mixture. Recording sessions occurred subsequent to the microstimulation behavioral sessions. Anesthetized subjects were positioned on the cage floor in the center of the calibrated environment. Frequency response characteristics of local field potentials (LFPs) and simultaneously recorded multi-unit clusters were determined from 15 logarithmically spaced pure tones ranging in frequency from $1 \mathrm{kHz}$ to $32 \mathrm{kHz}$ delivered at $70 \mathrm{~dB}$ SPL. Tone intervals were $100 \mathrm{~ms}$ on, $900 \mathrm{~ms}$ off. Each frequency was randomly repeated 48 times. All tones had a $5 \mathrm{~ms}$ cosine gated rise and fall time. 

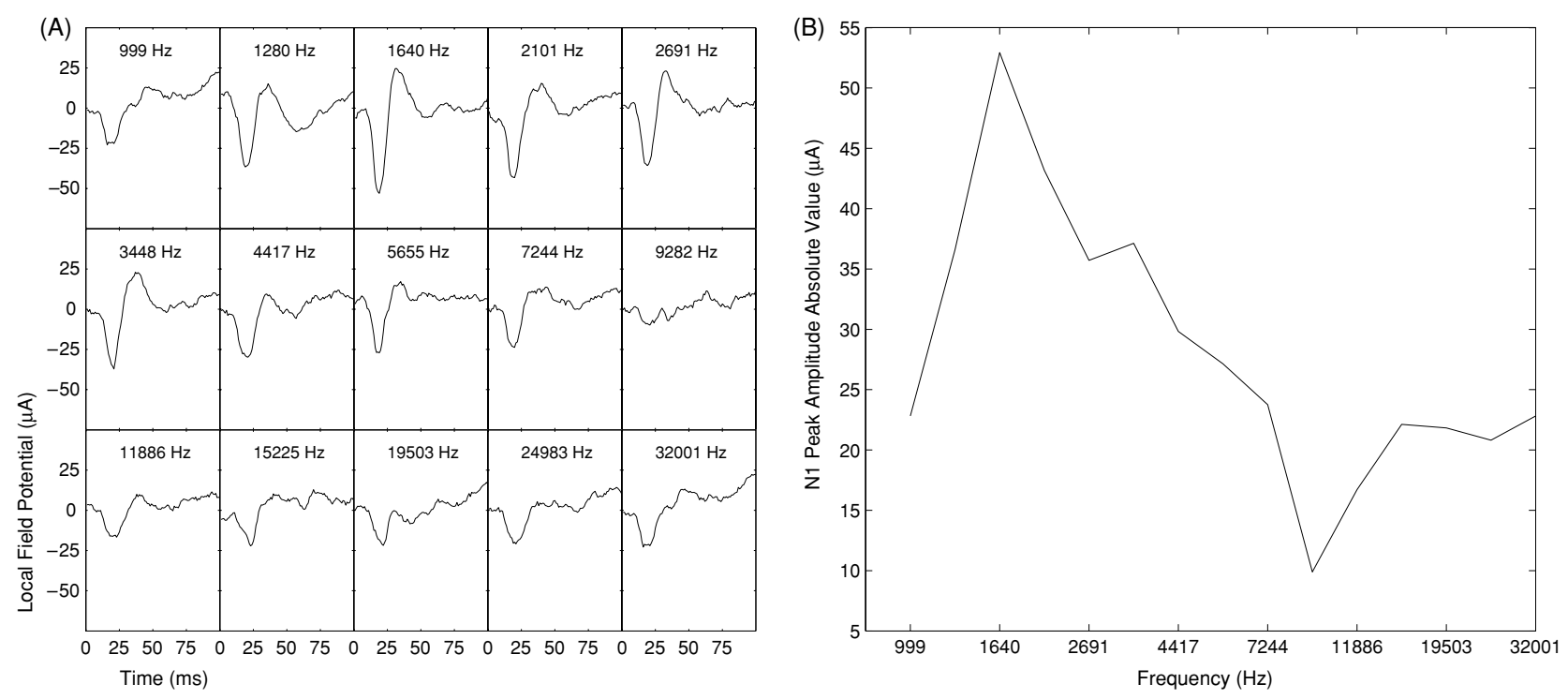

Figure 3. Acoustic frequency tuning from local field potentials in auditory cortex. (A) Fifteen separate 100 ms tonepips with frequencies logarithmically spaced from $1 \mathrm{kHz}$ to $32 \mathrm{kHz}$ were delivered at $70 \mathrm{~dB}$. The waveforms represent the average local field potential on a single electrode evoked by 48 repetitions of each tonepip stimulus. (B) The BF for this electrode from an N1 peak analysis was 1640 Hz.

Electrophysiological data were recorded on a multichannel acquisition processor (MAP) simultaneously for the 16 channels at $40 \mathrm{kHz}$ (Plexon Inc, Denison, TX). The data were filtered and amplified either for LFP recording $(1-300 \mathrm{~Hz}$, gain $=10000)$ or for multi-unit recording ( $250 \mathrm{~Hz}-8 \mathrm{kHz}$, programmable gain). The LFP responses were analyzed based on a previous study showing that the N1 peak of the LFP responses of the primary auditory cortex of guinea pig is tuned in the same way as single unit responses and exhibit this tuning over long-time periods (Galvan et al 2001). The LFP data from the first $50 \mathrm{~ms}$ after each stimulus onset were examined for auditory tuning. The LFP data were averaged for each of the fifteen tones and the frequency with the largest negative deflection from the $50 \mathrm{~ms}$ mean was determined to be the BF. When present on a recording electrode multi-unit responses were averaged as a function of the stimulus frequency, and the stimulus eliciting the largest response over the $100 \mathrm{~ms}$ tone duration was determined to be the multi-unit BF. When both LFP and multi-unit BF measurements were available on an electrode the overall $\mathrm{BF}$ of the electrode was determined as the average of the two measurements.

\section{Statistical evaluation of microstimulation-induced behavior}

In order to evaluate the behavior induced by cortical microstimulation predictions of the $\mathrm{BF}$ of each stimulated electrode were made based on the microstimulation-induced behavior. First the auditory generalization data were modeled for each subject by a logarithmic model. Then the predicted BF of the sensation could be calculated by evaluating this model at each behavioral data point. The predicted BFs for each stimulated electrode were plotted relative to the actual BF from the electrophysiological data and a correlation analysis was performed.

\section{Results}

In order to quantify microstimulation-induced behavior, both auditory-induced electrophysiological responses and auditoryinduced behavior characterization were characterized. The BFs across the electrode array were determined via analysis of auditory evoked LFP and multi-unit spike-rate fluctuations. Microstimulation-induced behavior was then evaluated relative to the natural auditory-evoked behavior to obtain a prediction of the sensation of the single electrode microstimulation. These data were evaluated relative to the electrophysiological BF recorded at each electrode.

\section{Auditory response electrophysiology}

Recording data consisted of LFP and multi-unit activity evoked by auditory stimuli across four, 16-channel microelectrode arrays implanted in the auditory cortex of the four behaviorally trained rats. An example of stimulus-triggered and averaged LFP data from a single electrode is shown in figure 3 . In this example, $1640 \mathrm{~Hz}$ produced the largest N1 peak deflection from the mean, and was determined to be the BF. The negative wave exhibits systematic tuning at frequencies neighboring $1640 \mathrm{~Hz}$.

$\mathrm{BF}$ data were evaluated for each subject for both the dorsal and ventral row of the array as shown in figure 4. From 64 channels across the four rats studied, LFP BFs were found from $999 \mathrm{~Hz}$ to $19503 \mathrm{~Hz}$, spanning nearly the entire spectrum used for auditory stimulation in this study. The multi-unit BFs spanned a similar range, from $1432 \mathrm{~Hz}$ to $21965 \mathrm{~Hz}$.

\section{A-A behavior results}

The subjects reliably performed the discrimination of the $1 \mathrm{kHz}$ and $16 \mathrm{kHz}$ frequencies. Example data from 14 sessions over 4 rats are shown in figure 5(A). Each column represents data 


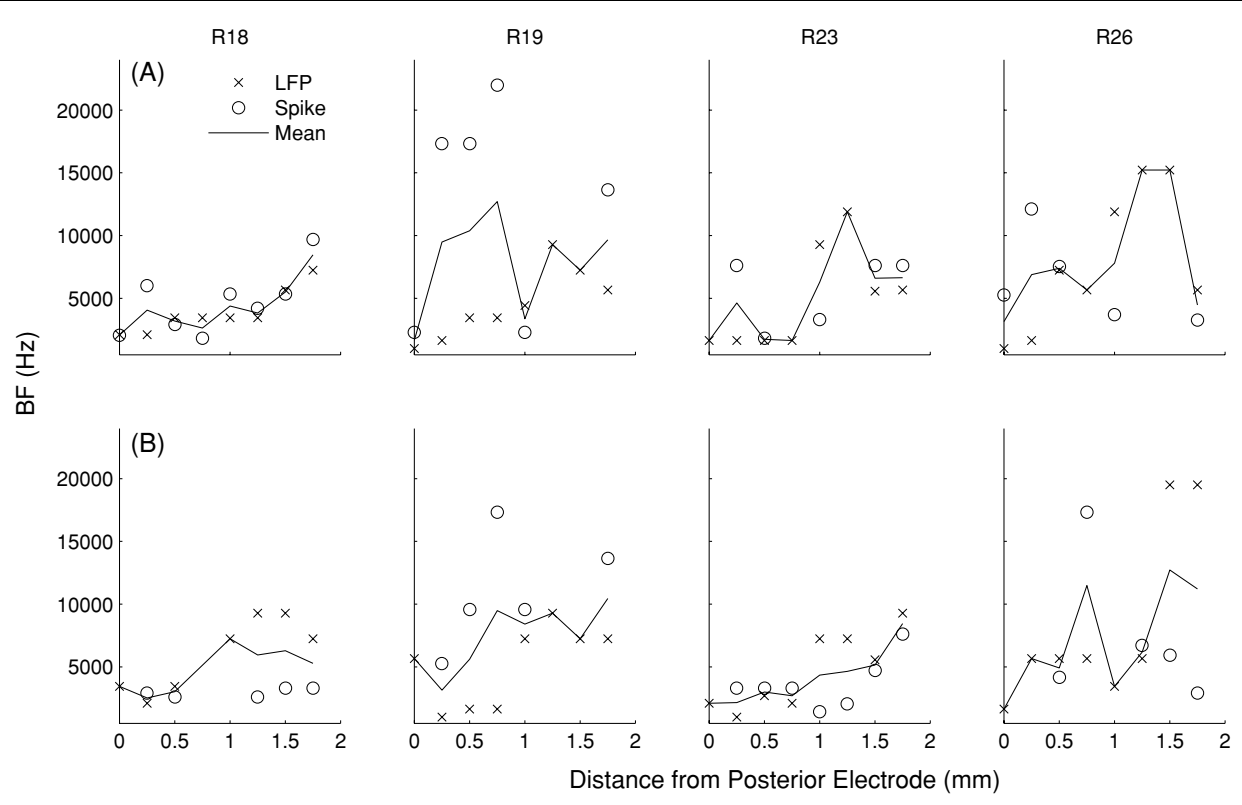

Figure 4. Best frequency maps in each of four rats. Local field potential and spike-rate best frequencies on the dorsal row (A) and ventral row (B) of the electrode array in each rat. The line represents the running mean of local field potential and spike-rate BF estimations. In general, $\mathrm{BF}$ increases as the anterior distance along the array increases.
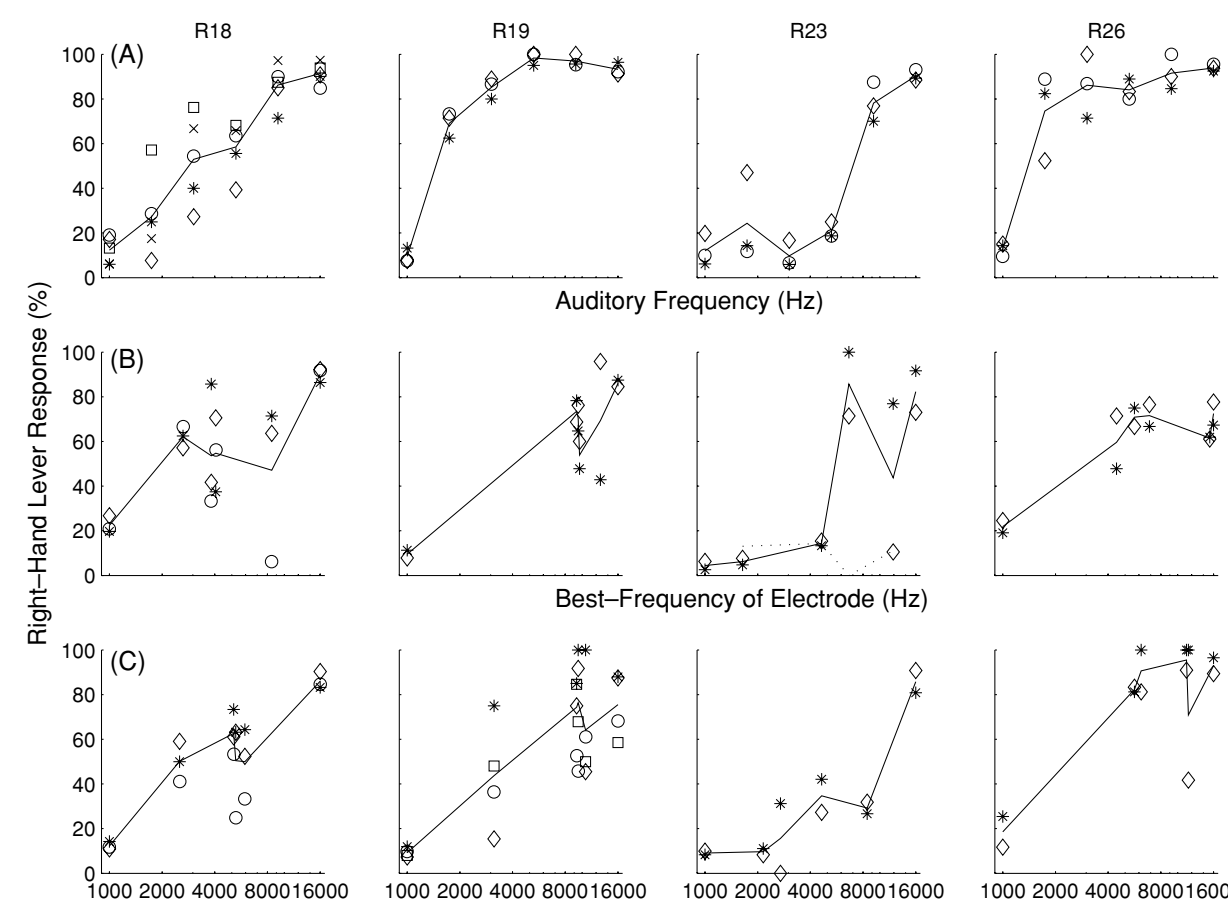

Auditory Frequency $(\mathrm{Hz})$
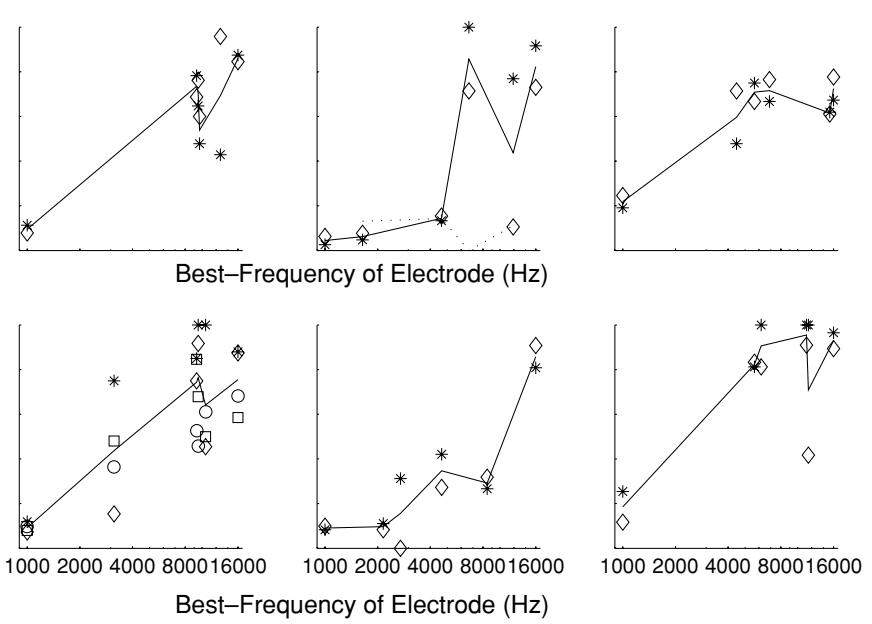

Figure 5. Auditory and microstimulation generalization curves. (A) Each marker type represents data from a single auditory generalization testing session plotted as a function of auditory stimulus frequency. Columns represent data from the four subjects. The ordinate represents the per cent of the responses on the right-hand lever. (B), (C) Microstimulation generalization curves from stimulation sessions on the dorsal row (B) or ventral row (C) of the electrode array, respectively. Subjects were rewarded for responding correctly to acoustic $1 \mathrm{kHz}$ and $16 \mathrm{kHz}$ tones. Microstimulation trials are plotted relative to the BF of the electrode used to provide the electrical stimulation. A control experiment using zero microstimulation current on probe trials is shown as the dotted line for the dorsal row (B) of subject R23.

from an individual subject. Daily sessions are represented by individual data markers, and the sliding mean of the sessions is indicated by the solid line. The overall performance of the discrimination task for these 14 sessions was $91.4 \pm 0.9 \%$ (mean \pm standard error). The rats consistently responded to auditory frequencies intermediate of the $1 \mathrm{kHz}$ and $16 \mathrm{kHz}$ reinforced frequencies. Across animals the response curves increased in a general monotonic fashion, such that higher 
frequency auditory cues resulted in more right-hand lever responses. There was a marked behavioral difference between subjects; however, the behavior within a subject was generally consistent across testing sessions.

\section{A-M behavior results}

Figures 5(B) and (C) show the A-M results plotted versus the $\mathrm{BF}$ of the electrode used for stimulation for the dorsal and ventral rows, respectively. Again, daily sessions are indicated by individual data markers, and the solid line indicates the sliding mean of the sessions. Overall, $20 \mathrm{~A}-\mathrm{M}$ sessions were performed across the four subjects. Microstimulation of the different electrodes resulted in similar lever-pressing behavior to the auditory trials. Microstimulation probe trials resulting in null responses were extremely rare (zero for 19 of the 20 sessions). Further, the response latency for microstimulation probe trials ( $1663 \pm 633 \mathrm{~ms}$; mean \pm standard deviation) was significantly shorter $(t$-test, $p<0.01)$ than the auditory probe trials $(2109 \pm 848 \mathrm{~ms} ;$ mean \pm standard deviation). These data suggest that microstimulation can provide a robust sensory cue for behavior.

A control experiment was conducted to ensure that the electrical stimulation apparatus provided no confounding behavioral cues (shown by the dotted line in figure 5(B) for subject R23). In this experiment, the session was conducted as a normal A-M session, except that the electrical stimulus isolator was turned off, ensuring that no microstimulation cues were delivered to the subject. Given the experimental design utilizing lever retraction and extension the subject readily responded on all trials; however, the subject responses to any of the control stimuli were not significantly different from each other ( $t$-test, $p<0.05)$. This result suggests that these trials provided no differential information to the subject. The low percentage of right-lever responses on these control trials is indicative of a left-lever bias for this subject.

To further evaluate the microstimulation-BF relationship, the data for all four subjects were evaluated cumulatively, as shown in figure 6 . Based on logarithmic fits to the auditory generalization data shown in figure 5(A), the microstimulation behavioral data were used to estimate the frequency of the induced sensation (predicted BF). These data were then plotted relative to the actual $\mathrm{BF}$ of each electrode, as determined by the electrophysiological measurements. Data from the dorsal and ventral row are depicted by circles and stars data markers, respectively. Correlation analysis of the cumulative predicted and actual BFs showed a significant positive correlation $(R=0.26, p<0.05)$. The cumulative ventral row data showed a stronger correlation $(R=0.40, p<0.01)$ than the dorsal row data $(R=0.12, p=0.54)$. The highest individual subject correlation was found for subject $\mathrm{R} 23(R=0.56, p<0.05)$.

\section{Discussion}

The objective of this study was to investigate the behavioral effects associated with microstimulation of the auditory cortex in the adult rat model. Subjects were required to behaviorally respond to cortical microstimulation probe trials randomly

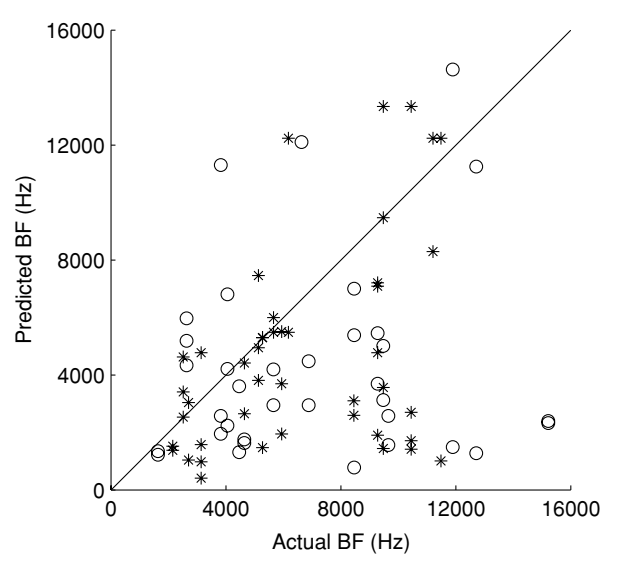

Figure 6. The relationship of the electrophysiologically determined $\mathrm{BF}$ to the behavioral BF. Based on a logarithmic model of the auditory generalization behavior, a predicted $\mathrm{BF}$ for the microstimulation trials was calculated. These data are positively correlated with the actual BF of each electrode as determined by local field potential and spike-rate measurements $(R=0.26$, $p<0.05)$.

inserted between trials of an auditory frequency discrimination task. The microstimulation-induced responses were analyzed relative to each microelectrode's auditory electrophysiology characteristics determined via LFP recording.

\section{The sensation basis of auditory cortex stimulation}

Animal stimuli-detection behavior in response to penetrating visual cortical stimulation was first evaluated by Bartlett and Doty (1980) in the primate and more recently by Troyk et al (2003) and Bradley et al (2005). Subsequently, Rousche and Normann (1999) assessed stimuli-detection behavior in response to auditory cortical stimulation first in the cat model and later in the rat model (Rousche et al 2003). These studies show that subjects readily respond to single electrode stimulation in a sensory detection paradigm. Recently, Scheich and Briendl (2002) evaluated penetrating auditory cortex stimulation in a discrimination paradigm in the Mongolian gerbil. The gerbils were able to discriminate spatial, temporal and spatio-temporal patterns of two-electrode stimulation. In this study we added to this literature, evaluating the spectral dependence of the auditory sensations evoked by single electrode microstimulation in different spatial locations within auditory cortex.

The results in figure 6 show that the basic spectral component of the elicited sensations was positively correlated with increasing BFs in the auditory cortex. This is consistent with visual cortex studies of systematic variation of sensation with microstimulation location. Both Brindley and Lewin (1968b) and Dobelle et al reported that surface visual cortex stimulation produced phosphenes that roughly corresponded to classical map expectations (Dobelle and Mladejovsky 1974).

\section{Temporal stimulation parameters}

The electrical stimulus pulse train used in this study was designed to match the temporal envelope of the auditory stimulus. Using surface stimulation of visual cortex, Dobelle 
reported 10-15 s of continuous stimulation before the elicited phosphenes faded (Dobelle and Mladejovsky 1974). Using penetrating stimulation, Schmidt et al (1996) reported a phosphene duration of $930 \mathrm{~ms}$ before the sensation faded. Dobelle employed $1 \mathrm{~s}$ pulse trains for surface auditory cortex stimulation, and did not publish any subject reports of the auditory sensation fading (Dobelle et al 1973). The electrical stimulation used in this study consisted of five $250 \mathrm{~ms}$ pulse trains (with $250 \mathrm{~ms}$ inter-train intervals). The durations of these pulse trains were well below the stimulus durations that led to fading sensation in the human studies. Therefore, it is likely that the sensation in this study persisted for the full 250 ms. Schmidt et al (1996) noted an accommodation to repeated stimulation that decreased the brightness of the phosphenes produced. Over 5 repetitions, the intensity dropped 20-30\%. Our stimulus trains may have resulted in accommodation, which likely would have decreased the loudness of the perceived sensation (Dobelle et al 1973).

The effect of temporal patterning of cortical microstimulation on sensation is still unknown. The $200 \mathrm{~Hz}$ bipolar stimulus used in this study would theoretically correspond to a $200 \mathrm{~Hz}$ amplitude-modulated sound; however, cortical neurons typically do not follow such fast modulation frequencies. The temporal patterning in this study was chosen to follow the paradigm used in other cortical microstimulation studies. However, it is noted that this abnormal temporal pattern in cortex may have contributed to some of the variability in the results. It is well documented that auditory cortex neurons exhibit 'on' responses to stimuli; however, whether this feature can be captured via microstimulation using a modulated pulse rate is not yet known. In primate somatosensory cortex, Romo et al (1998) reported identical behavioral responses to periodic and aperiodic cortical microstimulus trains of the same mean frequency. This suggests, at least for the train durations and stimulation modulation rates reported in that study, that aperiodic temporal information may not convey additional stimulus information.

\section{Spatial and temporal pattern of microstimulation}

Sensory stimulus encoding occurs in distributed spatial and temporal patterns (Chapin and Nicolelis 1999, Furukawa et al 2000, Villa and Abeles 1990). Given this characteristic, more sophisticated temporal and spatial microstimulation patterning is required for stimulus equivalency between natural auditory and electrical stimulation. The possibility of increasingly sophisticated temporal and spatial patterning of microstimulation is intimately tied to elegant device improvements. Micromachined device technologies offer intricate control over device parameters. Hetke et al (2003) demonstrated three-dimensional site spacing geometry in a cortical recording and stimulating device. The study presented here focused on the application basis of cortical microstimulation and thus utilized microwire arrays. However, several investigators in our laboratory have recently described success in utilizing complex micromachined devices for long-term cortical recording (Kipke et al 2003, Vetter et al 2004). These recent device developments indicate that complex spatial and temporal stimulation patterns in long-term preparations may be a possibility and provide a device framework for further cortical microstimulation neuroprosthetic research.

\section{Frequency discrimination and generalization}

Here we used a frequency discrimination and generalization behavioral task to determine the individual subjects' behavior relative to frequency. Although the speaker and testing box were calibrated at the position of the animal, an important possible confound exists in the relationship between frequency and intensity at the tympanic membrane. Thus, the animals may have utilized a combination of frequency and intensity to accomplish the discrimination. However, this strategy presumably would not have had an effect on the microstimulation trials, since the microstimulation was always provided at the same intensity.

The frequency generalization data for three of the four subjects exhibited a pronounced categorization shape rather than the characteristic sigmoidal shape. This may be due to an undersampling of the frequency domain near the subjects' point of subjective equality. Interestingly, the frequency at which the responses shifted from the left-hand lever to the right-hand lever was not identical for those three subjects. However, importantly, the behavior of the subjects over the course of multiple sessions was consistent, allowing a fairly robust determination of sensation based on behavior. This consistency was necessary to predict the spectral basis of the sensation evoked by microstimulation from the behavior.

\section{Electrophysiological recording and BF distribution}

This study determined auditory best frequencies under anesthesia. Recordings were not conducted in the awake state due to the small amplitudes of LFP recordings, and the recording artifact induced by movement; however, several studies have reported that BFs of single units do not change from awake to anesthetized states in rat auditory cortex (Gaese and Ostwald 2001, Talwar and Gerstein 2001). Several studies have described the complexity of auditory response properties in awake preparations (deCharms et al 1998, Gaese and Ostwald 2003), as well as the long-term dynamic nature of auditory cortex response properties (Witte et al 1999). These studies indicate that the BF-based analysis conducted here was valid, but also indicate that further studies are merited utilizing better devices and more thorough auditory cortical response measures in awake animal preparations.

Previous literature reports have shown that LFP recordings provide a robust measure of tuning across the electrode array, which is not significantly different from multiunit responses (Norena and Eggermont 2002). Our results generally show consistent tuning between LFP and multiunit responses; however, some of the electrode sites exhibited large differences between the LFP and multi-unit tuning. These differences are a possible confound in the microstimulation induced behavior results and may contribute to some of the unexplained behavioral variance. 
Recently, Galvan et al (2001) reported long-term frequency of tuning of LFPs in auditory cortex of guinea pig. Our recordings in auditory cortex of the rat produced tuning curves from $999 \mathrm{~Hz}$ to $24983 \mathrm{~Hz}$. This is slightly lower than the upper ranges of $\sim 40 \mathrm{kHz}$ found by Sally and Kelly (1988); however, most of the responses $>30 \mathrm{kHz}$ found by Sally and Kelly were proximal, or even anterior to the large anterior dorsoventral vessel in primary auditory cortex. Our electrode arrays were intentionally implanted significantly caudally of this vessel in order to avoid vascular injury. This may have biased our electrode placement in the lower frequency ranges. Additionally, the lower frequency representations of our rats may have been expanded due to training and microstimulationinduced plasticity, as discussed below.

\section{Cortical reorganization}

Due to the increasing potential failure of recording capabilities with lifetime of the microelectrode array, implantation did not occur until after the rats were successfully trained in the $1 \mathrm{kHz} / 16 \mathrm{kHz}$ discrimination task. It has been shown that behavioral training alters the representation of sensory stimuli in cortex (Bakin et al 1996, Recanzone et al 1992, 1993, Suga et al 2002). Furthermore, cortical microstimulation has been shown to change the functional organization of cortex (Chowdhury and Suga 2000, Dinse et al 1993, Maldonado and Gerstein 1996, Nudo et al 1990, Talwar and Gerstein 2001). In the current study, training was conducted for several months at $1 \mathrm{kHz}$ and $16 \mathrm{kHz}$. Additionally, several sessions of microstimulation were conducted before BF measurements were made. Our electrophysiological results show an overrepresentation of the lower frequencies, with $100 \%$ of the stimulated electrodes having BFs of $16 \mathrm{kHz}$ or lower.

The expanded cortical representations of the lower frequencies may have influenced the results of the microstimulation-induced behavior. The results from figure 6 show a lower correlation of microstimulation-induced behavior with $\mathrm{BF}$, relative to the correlation of the auditoryinduced behavior with frequency. If, in fact, the frequency representation within auditory cortex was distorted, this may have tended to diminish the strength of the BF-dependent microstimulation behavior and therefore may have contributed to some of the uncorrelated variance in the data.

In conclusion, the data suggest that cortical microstimulation in different locations in auditory cortex provides a behaviorally relevant auditory sensation. Furthermore, the behavior evoked by this sensation is dependent on the best frequency of the tissue local to the implanted electrode. In order to quantify the informationcarrying capacity of chronically implanted electrodes in the auditory cortex for a sensory neuroprosthesis, more parameters require investigation. Putatively, more knowledge of the sensations elicited by penetrating electrical stimulation of sensory cortex would allow for better engineering of the stimulus and electrodes to increase the information transfer. In our studies, the subject was not required to respond to other components of the stimulus (for example, bandwidth, background stimuli and temporal parameters); thus the sensation cannot be fully described. However, this study validates further exploration of the electrical stimulus perceptual parameters of penetrating auditory cortical stimulation.

\section{Acknowledgments}

We thank R Hurtado, N Langhals, L Good and J Williams for animal training and assistance with experiments, $\mathrm{C}$ Visser for electrode array fabrication, M Reilly, W Newsome, $P$ Killeen, and P Sabes for valuable feedback on experimental design, and R Witte, N Langhals, E Nunamaker, K Ludwig and L Huff-Brinkman for manuscript preparation assistance. This work was supported by the Defense Advanced Research Projects Agency (DARPA) under grants N66001-02-8059 and MDA972-00-1-0027 and NIH/NIBIB grant P41 EB00230-10.

\section{References}

Bakin J S, South D A and Weinberger N M 1996 Induction of receptive field plasticity in the auditory cortex of the guinea pig during instrumental avoidance conditioning Behav. Neurosci. 110 905-13

Bartlett J R and Doty R W 1980 An exploration of the ability of macaques to detect microstimulation of striate cortex Acta Neurobiol. Exp. $40713-27$

Bradley D C et al 2005 Visuotopic mapping through a multichannel stimulating implant in primate V1 J. Neurophysiol. 93 1659-70

Brindley G S and Lewin W S 1968a The sensations produced by electrical stimulation of the visual cortex J. Physiol. 196 479-93

Brindley G S and Lewin W S 1968b The visual sensations produced by electrical stimulation of the medial occipital cortex J. Physiol. 194 54-5

Chapin J K and Nicolelis M A L 1999 Principal component analysis of neuronal ensemble activity reveals multidimensional somatosensory representations J. Neurosci. Methods 94 121-40

Chowdhury S A and Suga N 2000 Reorganization of the frequency map of the auditory cortex evoked by cortical electrical stimulation in the big brown bat J. Neurophysiol. 83 1856-63

deCharms R C, Blake D T and Merzenich M M 1998 Optimizing sound features for cortical neurons Science 280 1439-43

Dinse H R, Recanzone G H and Merzenich M M 1993 Alterations in correlated activity parallel ICMS-induced representational plasticity Neuroreport 5 173-6

Dobelle W H and Mladejovsky M G 1974 Phosphenes produced by electrical stimulation of human occipital cortex, and their application to the development of a prosthesis for the blind J. Physiol. 243 553-76

Dobelle W H, Stensaas S S, Mladejovsky M G and Smith J B 1973 A prosthesis for the deaf based on cortical stimulation Ann. Otol. Rhinol. Laryngol. 82 445-63

Furukawa S, Xu L and Middlebrooks J C 2000 Coding of sound-source location by ensembles of cortical neurons J. Neurosci. 20 1216-28

Gaese B H and Ostwald J 2001 Anesthesia changes frequency tuning of neurons in the rat primary auditory cortex J. Neurophysiol. 86 1062-6

Gaese B H and Ostwald J 2003 Complexity and temporal dynamics of frequency coding in the awake rat auditory cortex Eur. J. Neurosci. 18 2638-52

Galvan V V, Chen J and Weinberger N M 2001 Long-term frequency tuning of local field potentials in the auditory cortex of the waking guinea pig J. Assoc. Res. Otolaryngol. 2 199-215 
Groh J M, Born R T and Newsome W T 1997 How is a sensory map read out? Effects of microstimulation in visual area Mt on saccades and smooth pursuit eye movements J. Neurosci. 17 4312-30

Hetke J F, Williams J C, Pellinen D S, Vetter R J and Kipke D R 2003 3-D silicon probe array with hybrid polymer interconnect for chronic cortical recording 1st Int. IEEE EMBS Conf. on Neural Engineering (Capri, Italy) pp 181-4

Kilgard M P and Merzenich M M 1999 Distributed representation of spectral and temporal information in rat primary auditory cortex Hear Res. 134 16-28

Kipke D R, Vetter R J, Williams J C and Hetke J F 2003 Silicon-substrate intracortical microelectrode arrays for long-term recording of neuronal spike activity in cerebral cortex IEEE Trans. Neur. Syst. Reh. 11 151-5

Maldonado P E and Gerstein G L 1996 Reorganization in the auditory cortex of the rat induced by intracortical microstimulation: a multiple single-unit study Exp. Brain Res. $112420-30$

Norena A and Eggermont J J 2002 Comparison between local field potentials and unit cluster activity in primary auditory cortex and anterior auditory field in the cat Hear Res. 166 $202-13$

Nudo R J, Jenkins W M and Merzenich M M 1990 Repetitive microstimulation alters the cortical representation of movements in adult rats Somatosens. Mot. Res. 7 463-83

Nunez P L 1981 Electric Fields of the Brain: the Neurophysics of $E E G$ (New York: Oxford University Press)

Penfield W and Perot P 1963 The brain's record of auditory and visual experience Brain 86 595-696

Penfield W and Rasmussen T 1950 The Cerebral Cortex of Man (New York: Macmillan)

Recanzone G H, Merzenich M M and Schreiner C E 1992 Changes in the distributed temporal response properties of $\mathrm{Si}$ cortical neurons reflect improvements in performance on a temporally based tactile discrimination task J. Neurophysiol. 67 1071-91

Recanzone G H, Schreiner C E and Merzenich M M 1993 Plasticity in the frequency representation of primary auditory cortex following discrimination training in adult owl monkeys J. Neurosci. 13 87-103

Romo R, Hernandez A, Zainos A, Brody C D and Lemus L 2000 Sensing without touching: psychophysical performance based on cortical microstimulation Neuron $26273-8$

Romo R, Hernandez A, Zainos A and Salinas E 1998 Somatosensory discrimination based on cortical microstimulation Nature 392 387-90

Ronner S F, Foote W E and Pollen D A 1981 Intracortical microstimulation of neurons in the visual cortex of the cat Electroencephalogr. Clin. Neurophysiol. 52 375-7

Rousche P J and Normann R A 1999 Chronic intracortical microstimulation (ICMS) of cat sensory cortex using the Utah intracortical electrode array IEEE Trans. Rehabil. Eng. $756-68$

Rousche P J, Otto K J and Kipke D R 2001 Intracortical Microstimulation (ICMS) in Rat Auditory Cortex: Examination of Relationship between Pure Tone and ICMS-evoked
Generalization Gradient Curves (San Diego, CA Society for Neuroscience) (abstract)

Rousche P J, Otto K J, Reilly M P and Kipke D R 2003 Single electrode micro-stimulation of rat auditory cortex: an evaluation of behavioral performance Hear Res. 179 62-71

Sally S L and Kelly J B 1988 Organization of auditory cortex in the albino rat: sound frequency J. Neurophysiol. 59 1627-38

Salzman C D, Britten K H and Newsome W T 1990 Cortical microstimulation influences perceptual judgments of motion direction Nature 346 174-7

Salzman C D, Murasugi C M, Britten K H and Newsome W T 1992 Microstimulation in visual area Mt: effects on direction discrimination performance $J$. Neurosci. 12 2331-55

Scheich H and Breindl A 2002 An animal model of auditory cortex prostheses Audiol Neuro-Otol. 7 191-4

Schmidt E M, Bak M J, Hambrecht F T, Kufta C V, O'Rourke D K and Vallabhanath P 1996 Feasibility of a visual prosthesis for the blind based on intracortical microstimulation of the visual cortex Brain 119 507-22

Seidemann E, Zohary E and Newsome W T 1998 Temporal gating of neural signals during performance of a visual discrimination task Nature $39472-5$

Stoney S D, Thompson W D and Asanuma H 1968 Excitation of pyramidal tract cells by intracortical microstimulation: effective extent of stimulating current $J$. Neurophysiol. 31 659-69

Suga N, Xiao Z, Ma X and Ji W 2002 Plasticity and corticofugal modulation for hearing in adult animals Neuron 36 9-18

Talwar S K and Gerstein G L 2001 Reorganization in awake rat auditory cortex by local microstimulation and its effect on frequency-discrimination behavior J. Neurophysiol. 86 $1555-72$

Tehovnik E J 1996 Electrical stimulation of neural tissue to evoke behavioral responses J. Neurosci. Methods 65 1-17

Troyk P, Bak M J, Berg J, Bradley D, Cogan S E, Erickson R, Kufta C V, Mccreery D B, Schmidt E M and Towle V 2003 A model for intracortical visual prosthesis research Artif. Organs 27 1005-15

Vetter R J, Williams J C, Hetke J F, Nunamaker E A and Kipke D R 2004 Chronic neural recording using silicon-substrate microelectrode arrays implanted in cerebral cortex IEEE Trans. Biomed. Eng. 51 896-904

Villa A E and Abeles M 1990 Evidence for spatiotemporal firing patterns within the auditory thalamus of the cat Brain Res. 509 325-27

Williams J C, Rennaker R L and Kipke D R 1999 Long-term neural recording characteristics of wire microelectrode arrays implanted in cerebral cortex Brain Res. Protocols 4 303-13

Witte R S, Otto K J, Williams J C and Kipke D R 1999 Pursuing dynamic reorganization in auditory cortex using chronic multichannel unit recordings in awake, behaving cats Neurocomputing 26-7 593-600

Woody C D and Engel J Jr 1972 Changes in unit activity and thresholds to electrical microstimulation at coronal-pericruciate cortex of cat with classical conditioning of different facial movements J. Neurophysiol. 35 230-41 